\title{
Brustgesundheitszentrum Tirol, Universitätskliniken, Innsbruck
}

\author{
Christian Marth Thomas Frede Elisabeth Müller-Holzner Susanne Taucher \\ Günter Daxenbichler Irene Virgolini Günther Gastl Peter Lukas Thomas Schöller \\ Gottfried Wechselberger Dieter zur Nedden \\ Brustgesundheitszentrum Tirol, Innsbruck, Österreich
}

Es ist einsichtig, dass die Behandlung des Mammakarzinoms eine interdisziplinäre Vorgangsweise erfordert. Das $\mathrm{Zu}$ sammenspiel der verschiedenen Fachdisziplinen funktioniert erfahrungsgemäß nur dann gut und reibungslos, wenn eine gewisse örtliche Nähe gegeben und den Beteiligten die Zugehörigkeit zu einem Programm bewusst ist. Das Brustgesundheitszentrum Tirol ist an der Universitätsklinik Innsbruck etabliert. Eine sehr günstige Voraussetzung ist dadurch gegeben, dass die zentralen Säulen eines Brustzentrums, Radiologie, Pathologie, Brustambulanz, Operationssäle und Bettenstation unter einem Dach vereint sind. Als eine zweite wichtige Voraussetzung für die Funktion und Akzeptanz des Brustzentrums wird der respektvolle und freundschaftliche Umgang der Kollegen aus den verschiedenen Disziplinen, untereinander und mit dem Pflegepersonal und den Patienten gesehen.

Der hohe Standard der medizinischen Betreuung mit Auswirkungen auf die Heilungs- und Überlebensraten der Patientinnen, die effektive Ausnutzung der Ressourcen und viele andere Qualitätsmerkmale für eine möglichst optimale Behandlung von Brustkrebspatientinnen, die am besten in einem zertifizierten Brustzentrum erreichbar sind, wurden in zahlreichen Abhandlungen bereits dargelegt. Es soll dies hier nicht noch einmal wiederholt werden. Wir möchten uns bei der Darstellung unseres Brustgesundheitszentrums auf einen Punkt konzentrieren: Das Brustzentrum als eine Institution, welche angewandte Forschung auf dem Gebiete des Mammakarzinoms ermöglicht und unterstützt.

Die wichtigsten Instrumente und Einrichtungen, die uns ermöglichen sollen, einen relevanten Beitrag zur wissenschaftlichen Forschung zu machen, sind das morphologische Labor, das biochemische Labor, die Gewebebank, die Serumbank, die Studienzentrale und die onkologische Datenbank.

Unser morphologisches Labor wird von einer Pathologin geleitet, der Wissenschaft ein hohes Anliegen ist. Dies betrifft die kritische Evaluierung des täglichen Tuns, z.B. der histologischen Analyse von Stanzbiopsien mittels Gefrierschnitten, der akribischen Aufarbeitung von Lymphknoten usw., aber auch die Zurverfügungstellung von Laborkapazität und -knowhow für wissenschaftliche Projekte. Ein äußerst geschätzer Beitrag dieses Labors zur Verwirklichung von Forschungsprojekten sind die Erstellung von Tissue Arrays und vor allem die «Fütterung» und Pflege der Gewebebank.

Die Etablierung einer Gewebebank geht zurück auf das Jahr 1986, d.h. vor nunmehr 20 Jahren wurde begonnen, Gewebe systematisch und möglichst vollzählig zu sammen und in gefrorenem Zustand zu lagern. Sowohl das Sammeln als auch die Gewinnung von Gewebsaliquoten in gefrorenem Zustand für Untersuchungen sind mit logistischen und und technischen Problemen behaftet. Um einer Degradierung, insbesondere von RNA vorzubeugen, muss das Gewebe, einem Schnellschnitt gleich, sofort ins Labor gebracht und dort sofort beurteilt und schockgefroren werden. Durch genaue Anleitung (SOP), Erziehung und einem effizienten Fehlermanagement funktioniert dies zufriedenstellend. Als ein sehr geschätzes Service pulverisieren wir das Gewebe, ein technisch anspruchsvoller Vorgang, sodass identische Aliquote mühelos entnommen werden können. Die Tumorbank enthält derzeit 6000 Gewebsproben von Patientinnen mit gynäkologischen und Mammakarzinomen, gutartigen Tumoren dieser Bereiche und Körperflüssigkeiten.

Das biochemische Labor ist eine Serviceeinrichtung, die molekularbiologische und biochemische Analysen für wissenschaftliche Projekte ermöglicht, mit entsprechender Ausrüstung und Knowhow. Seit 20 Jahren wird bei jeder Tumormarkerbestimmung Serum in mehreren Aliquoten tiefgefroren. Die Serumbank enthält mittlerweile ca. 400000 Serumproben von ca. 170000 Blutabnahmen bei ca. 8000-10 000 Patientinnen mit Mamma- oder Genitalkarzinomen. Die Labor-EDV zusammen mit der Tumordatenbank erlaubt

\begin{tabular}{ll}
\hline KARGER & @ 2006 S. Karger GmbH, Freiburg \\
Fax +4976145207 14 & Accessible online at: \\
$\begin{array}{l}\text { E-mail Information@Karger.de } \\
\text { www.karger.com }\end{array}$ & www.karger.com/brc
\end{tabular}

Prof. Dr. Christian Marth

Universitätsklinik für Frauenheilkunde

Medizinische Universität Innsbruck

Anichstrasse 35, 6020 Innsbruck, Österreich

Tel. +4351250423050

E-Mail christian.marth@uibk.ac.at 
ein leichtes Aussuchen und Heraussuchen der Proben. Die technische Voraussetzung ist durch eine große Gefrieranlage gegeben.

Klinische Forschung erfordert die Teilnahme an MulticenterStudien. Der Kliniker ist neben seiner ärztlichen Tätigkeit kaum in der Lage, alle Ein-und Ausschlusskriterien mehrerer Studien im Kopf zu haben und insbesondere die aufwendige Dokumentation und Kommunikation zu bewältigen. Aus diesem Grunde wurde in unserem Brustzentrum eine Studienzentrale etabliert. Diese wird von einer Person (Akademikerstelle, ganztägig) betreut und verwaltet derzeit 21 Studien. Eine zusätzliche Study Nurse ist beantragt.

Auch die Tumordatenbank ist eine Einrichtung, ohne die ernst- hafte klinisch orientierte Forschung (translational research) nicht machbar ist. Die Daten werden aus veschiedenen Datenbanken (Krankenhausinformationssystem, Labor-EDV, lokale EDV-Systeme an anderen Abteilungen) extrahiert und nach Plausibilitätsprüfung in die Tumordatenbank eingebracht. Eine Ärztin ist ganztägig mit der Übernahme, Eingabe und Aktualisierung der Daten ausgelastet.

Die analytischen Möglichkeiten der beiden Labors, die Gewebe- und Serumdatenbank, die Studienzentrale und die Tumordatenbank stellen zusammen eine äußerst wertvolle und geschätze Basis für wissenschaftliche Forschung dar. Diese Instrumente machen unser Brustzentrum auch für multizentrische und interdisziplinäre Forschungsprojekte attraktiv. 